\title{
INEQUALITIES FOR THE FIRST AND SECOND DERIVATIVES OF ALGEBRAIC POLYNOMIALS ON AN ELLIPSE
}

\author{
Tatiana M. Nikiforova ${ }^{1,2, *}$
}

\footnotetext{
${ }^{1}$ N. N. Krasovskii Institute of Mathematics and Mechanics of the Ural Branch of the Russian Academy of Sciences 16 S. Kovalevskaya Str., Yekaterinburg, Russia

${ }^{2}$ Ural Federal University 19 Mira Str., Yekaterinburg, Russia

Communicated by Szilárd Gy. Révész
}

\section{Original Research Paper}

Received: Oct 8, 2021 - Accepted: Oct 21, 2021

First published online: November 8, 2021

ㄷ 2021 The Author(s)

\section{ABSTRACT}

We prove a theorem on the preservation of inequalities between functions of a special form after differentiation on an ellipse. In particular, we obtain generalizations of the Duffin-Schaeffer inequality and the Vidensky inequality for the first and second derivatives of algebraic polynomials to an ellipse.

\section{KEYWORDS}

Polynomial, derivative, ellipse, majorant, Bernstein inequality, Duffin-Schaeffer inequality, Vidensky inequality

\section{MATHEMATICS SUBJECT CLASSIFICATION (2020)}

Primary 41A17; Secondary 30A10, 30C10

\section{INTRODUCTION}

The next result called the Bernstein inequality is famous.

THEOREM 1.1. Let $\tau_{n}$ be a trigonometric polynomial of degree $n$ with complex coefficients such that

$$
\left|\tau_{n}(t)\right| \leq 1, \quad t \in \mathbb{R} .
$$

Then

$$
\left|\tau_{n}^{\prime}(t)\right| \leq n, \quad t \in \mathbb{R} .
$$

In 1912, S. N. Bernstein [1] proved (1.1) for real even and odd trigonometric polynomials with the constant $n$ and with $2 n$ in general case. E. Landau pointed out simple trick for obtaining the constant $n$ from Bernstein's result. Soon a lot of different proofs of (1.1) appeared. One of the most general due to M. Riesz [6].

\footnotetext{
${ }^{*}$ Corresponding author. E-mail: t.m.nikiforova@yandex.ru
} 
Theorem 1.1 implies that if an algebraic polynomial $p_{n}$ of degree $n$ with complex coefficients satisfies

$$
\left|p_{n}(x)\right| \leq 1=\left|T_{n}(x)+\frac{i}{n} \sqrt{1-x^{2}} T_{n}^{\prime}(x)\right|, \quad x \in(-1,1),
$$

where

$$
T_{n}(x)=\frac{\left(x+\sqrt{x^{2}-1}\right)^{n}+\left(x-\sqrt{x^{2}-1}\right)^{n}}{2}
$$

is the Chebyshev polynomial of the first kind, then

$$
\left|p_{n}^{\prime}(x)\right| \leq \frac{n}{\sqrt{1-x^{2}}}=\left|T_{n}^{\prime}(x)+\left(\frac{i}{n} \sqrt{1-x^{2}} T_{n}^{\prime}(x)\right)^{\prime}\right|, \quad x \in(-1,1) .
$$

Inequality (1.3) is also called the Bernstein inequality.

In 1938, R. J. Duffin and A. C. Schaeffer [5] got a new simplified proof of the Markov inequality for algebraic polynomials on an interval. An important point in their proof is the extension of (1.3) to higher derivatives. Namely if $p_{n}$ satisfies (1), then for all $k=1, \ldots, n$

$$
\left|p_{n}^{(k)}(x)\right| \leq\left|T_{n}^{(k)}(x)+\left(\frac{i}{n} \sqrt{1-x^{2}} T_{n}^{\prime}(x)\right)^{(k)}\right|, \quad x \in(-1,1) .
$$

This result is called the Duffin-Schaeffer inequality.

In 1951, V. S. Vidensky introduced majorants of the form $K_{n}(x)+i \sqrt{1-x^{2}} L_{n-1}(x)$, where $K_{n}$ and $L_{n-1}$ are algebraic polynomials of degrees $n$ and $n-1$ respectively such that all the zeros of $K_{n}$ and $L_{n-1}$ lie on $[-1,1]$ and separate one another. He proved the following generalization of the Duffin-Schaeffer inequality.

THEOREM 1.2. For any algebraic polynomial $p_{n}$ of degree $n$ such that

$$
\left|p_{n}(x)\right| \leq\left|K_{n}(x)+i \sqrt{1-x^{2}} L_{n-1}(x)\right|, \quad x \in(-1,1) .
$$

the following inequalities hold

$$
\left|p_{n}^{(k)}(x)\right| \leq\left|K_{n}^{(k)}(x)+i\left(\sqrt{1-x^{2}} L_{n-1}(x)\right)^{(k)}\right|, \quad x \in(-1,1), k=1, \ldots, n .
$$

Bernstein also obtained pointwise inequalities for derivatives of algebraic polynomials on the unit circle [3] and on the real axis [2, p. 56].

THEOREM 1.3. Let $p_{n}$ and $H_{n}$ be algebraic polynomials of degree at most $n$ and assume that all the zeros of $H_{n}$ lie in the open unit disk. Then the inequality

$$
\left|p_{n}(z)\right| \leq\left|H_{n}(z)\right|, \quad|z|=1,
$$

implies

$$
\left|p_{n}^{(k)}(z)\right| \leq\left|H_{n}^{(k)}(z)\right|, \quad|z|=1, k \in \mathbb{N} .
$$

THEOREM 1.4. Let $p_{n}$ and $H_{n}$ be algebraic polynomials of degree at most $n$ and assume that all the zeros of $H_{n}$ lie in the upper half-plane. If

$$
\left|p_{n}(x)\right| \leq\left|H_{n}(x)\right|, \quad x \in \mathbb{R},
$$

then

$$
\left|p_{n}^{(k)}(x)\right| \leq\left|H_{n}^{(k)}(x)\right|, \quad x \in \mathbb{R}, k \in \mathbb{N} .
$$

The connection between these results is interesting. Bernstein proved Theorem 1.3 by using Theorem 1.4. Moreover, Theorem 1.3 yields Theorem 1.1.

In 1947, De Bruijn [4] extended Theorem 1.3 to an arbitrary convex domain. 
Let us denote by $G$ the Joukowsky transform $G(w)=\frac{1}{2}\left(w+\frac{1}{w}\right)$. Fix $r \in(0,1]$ and consider the set

$$
E_{r}=\left\{z=G(r w)=\frac{1}{2}\left(r w+\frac{1}{r w}\right),|w|=1\right\} .
$$

The set $E_{r}$ is an ellipse for $r \in(0,1)$ and is the segment $[-1,1]$ for $r=1$. An ellipse is a natural generalization of a segment and a circle. However, methods of proving Theorems 1.2 and 1.3 for a segment and a circle are not directly applicable to an ellipse.

In 1937, W. E. Sewell [7], using (1.1), obtained an estimate of the derivative of an algebraic polynomial on an ellipse.

THEOREM 1.5. For any algebraic polynomial $p_{n}$ of degree $n$ such that

$$
\left|p_{n}(z)\right| \leq 1, \quad z \in E_{r}
$$

the following inequality holds

$$
\left|p_{n}^{\prime}(z)\right| \leq \frac{2 n}{1 / r-r}, \quad z \in E_{r} .
$$

Moreover, Sewell's reasoning gives an ellipse version of Bernstein's inequality (1.3): if (1.4) holds, then

$$
\left|p_{n}^{\prime}(z)\right| \leq \frac{n}{\sqrt{\left(r^{2}+1 / r^{2}\right) / 2-|z|^{2}}}, \quad z \in E_{r} .
$$

In this paper we prove the following theorem.

THEOREM 1.6. Suppose that a function $M_{n}(z), z \in E_{r}$, has the form

$$
M_{n}(G(r w))=\frac{R_{2 n}(w)}{w^{n}}, \quad|w|=1,
$$

where $R_{2 n}$ is an algebraic polynomial of degree $n$ having all $2 n$ zeros in the open unit disk. If an algebraic polynomial $p_{n}$ of degree at most $n$ satisfies

$$
\left|p_{n}(z)\right| \leq\left|M_{n}(z)\right|, \quad z \in E_{r},
$$

then for $k=1,2$

$$
\left|p_{n}^{(k)}(z)\right| \leq\left|M_{n}^{(k)}(z)\right|, \quad z \in E_{r} .
$$

For $M_{n}(G(r w))=w^{n}$ and $k=1$ inequalities (1.8) and (1.9) turn into Sewell's inequalities (1.4) and (1.6). We show this in Section 4. Also, Theorem 1.6 can be viewed as a generalization of the Duffin-Schaeffer and the Vidensky inequalities to the ellipse for the first and second derivatives of algebraic polynomials.

\section{PRELIMINARIES}

Denote the inverse of the Joukowsky transform by $h(z)=z+\sqrt{z^{2}-1}$. Its continuous single-valued branches map $E_{r}$ to the circles

$$
|z|=r \text { and }|z|=1 / r
$$

depending on the choice of the branch of $\sqrt{z^{2}-1}$. By $\sqrt{z^{2}-1}$ we shall mean that branch of $\sqrt{z^{2}-1}$, for which $h$ maps $E_{r}$ to $|z|=r$. Then the function

$$
w(z)=\frac{1}{r} h(z)=\frac{1}{r}\left(z+\sqrt{z^{2}-1}\right)
$$

maps $E_{r}$ on the unit circle, its inverse is $z=G(r w)=\frac{1}{2}\left(r w+\frac{1}{r w}\right)$. We have

$$
\sqrt{(G(r w))^{2}-1}=r w-G(r w)=\frac{1}{2}\left(r w-\frac{1}{r w}\right) \text {. }
$$


Note that

$$
\frac{d w}{d z}=\frac{1}{z_{w}^{\prime}}=\frac{1}{(G(r w))^{\prime}}=\frac{2 w}{r w-1 /(r w)}=\frac{w}{\sqrt{(G(r w))^{2}-1}}, \quad|w|=1 .
$$

Let us consider functions $f(z), z \in E_{r}$, having the form

$$
f_{n}(G(r w))=\frac{Q_{2 n}(w)}{w^{n}}, \quad|w|=1,
$$

where $Q_{2 n}$ is an algebraic polynomial of degree at most $2 n$. We say that the polynomial $Q_{2 n}$ corresponds to $f_{n}$ on the unit circle. In particular, an algebraic polynomial $P(z)$ of degree at most $n$ on $E_{r}$ belongs to this class of functions. Moreover, the functions

$$
f_{n}(z)=K_{n}(z)+\sqrt{z^{2}-1} L_{n-1}(z), \quad z \in E_{r},
$$

where $K_{n}, L_{n-1}$ are algebraic polynomials of degrees $n$ and $n-1$ respectively have the form (2). This can be shown by substitutions $z=G(r w)$ and (2.1).

Recall that Szegö's composition of two polynomials $Q(z)=\sum_{j=0}^{n}\left(\begin{array}{l}n \\ j\end{array}\right) q_{j} z^{j}$ and $H(z)=\sum_{j=0}^{n}\left(\begin{array}{l}n \\ j\end{array}\right) h_{j} z^{j}$ of degree $n$ is given by

$$
Q * H(z)=\sum_{j=0}^{n}\left(\begin{array}{l}
n \\
j
\end{array}\right) q_{j} h_{j} z^{j}
$$

In 1922, G. Szegő [8] proved the following theorem.

THEOREM 2.1. If all the zeros of $Q$ lie in a circular region $C$, then every zero of $Q * H$ has the form $-\alpha \beta$, where $\alpha$ is a suitably chosen point in $C$ and $\beta$ is a zero of $H$.

Let us check that

$$
z Q^{\prime}(z)-\frac{n}{2} Q(z)=Q * D_{n}(z)
$$

where $D_{n}(z)=\frac{n}{2}(z+1)^{n-1}(z-1)$. Indeed, $Q(z)=Q * I_{n}(z)$, where $I_{n}(z)=(z+1)^{n}$. For $z Q^{\prime}(z)$ we have

$$
\begin{gathered}
z Q^{\prime}(z)=\sum_{j=1}^{n}\left(\begin{array}{l}
n \\
j
\end{array}\right) q_{j} j z^{j}=Q *\left(\sum_{j=1}^{n}\left(\begin{array}{l}
n \\
j
\end{array}\right) j z^{j}\right) \\
=Q * z\left(\sum_{j=0}^{n}\left(\begin{array}{l}
n \\
j
\end{array}\right) z^{j}\right)^{\prime}=Q * z\left((z+1)^{n}\right)^{\prime}=Q * n z(z+1)^{n-1} .
\end{gathered}
$$

Hence

$$
z Q^{\prime}(z)-\frac{n}{2} Q(z)=\left[Q * n z(z+1)^{n-1}\right]-\frac{n}{2}\left[Q *(z+1)^{n}\right]=Q *\left(\frac{n}{2}(z+1)^{n-1}(z-1)\right) .
$$

\section{PROOF OF THEOREM 1.5}

Proof. Let us prove (1.9) for the first derivative. For $|\alpha|<1$ we consider the difference $f_{n}(z)=$ $M_{n}(z)-\alpha p_{n}(z)$. It can be represented as

$$
f_{n}(z(w))=\frac{Q_{2 n}(w)}{w^{n}}, \quad|w|=1,
$$

where $Q_{2 n}$ is an algebraic polynomial of degree at most $2 n$. Inequality (1.8) and Rouche's theorem imply that $Q_{2 n}$ has all $2 n$ zeros in the open unit disk. Using (2.2) and (2.3), we get

$$
\begin{aligned}
\frac{d f_{n}}{d z} & =\frac{d\left(Q_{2 n} / w^{n}\right)}{d w} \cdot \frac{d w}{d z}=\frac{Q_{2 n}^{\prime} w^{n}-Q_{2 n} n w^{n-1}}{w^{2 n}} \frac{2 w}{r w-1 /(r w)} \\
& =\frac{2\left(Q_{2 n}^{\prime} w-n Q_{2 n}\right)}{w^{n}(r w-1 /(r w))}=\frac{2\left(Q_{2 n} * D_{2 n}\right)}{w^{n}(r w-1 /(r w))},
\end{aligned}
$$


where $D_{2 n}(u)=n(u+1)^{2 n-1}(u-1)$. By Szegö's theorem 2.1, the numerator of (3.1) has all the zeros in the open unit disk. Therefore,

$$
f_{n}^{\prime}(z)=M_{n}^{\prime}(z)-\alpha p_{n}^{\prime}(z) \neq 0, \quad z \in E_{r}
$$

To obtain a contradiction, suppose that $\left|M_{n}^{\prime}\left(z_{0}\right)\right|<\left|p_{n}^{\prime}\left(z_{0}\right)\right|$ at some point $z_{0} \in E_{r}$. Take $\alpha_{0}=\frac{M_{n}^{\prime}\left(z_{0}\right)}{p_{n}^{\prime}\left(z_{0}\right)}$, then $\left|\alpha_{0}\right|<1$ and we have $M_{n}^{\prime}\left(z_{0}\right)-\alpha_{0} p_{n}^{\prime}\left(z_{0}\right)=0$ contrary to (3.2).

We now prove (1.9) for the second derivative. This case can be handled in much the same way, the only difference being in the analysis of $f_{n}^{\prime \prime}(z)=M_{n}^{\prime \prime}(z)-\alpha p_{n}^{\prime \prime}(z), z \in E_{r}$. Set $S_{2 n}=2\left(Q_{2 n} * D_{2 n}\right)$. We have

$$
\frac{d^{2} f_{n}}{d z^{2}}=\frac{d}{d w} \frac{S_{2 n}}{w^{n}(r w-1 /(r w))} \frac{d w}{d z}=2 \frac{S_{2 n}^{\prime} w-S_{2 n} n-S_{2 n} \frac{(r w)^{2}+1}{(r w)^{2}-1}}{w^{n}(r w-1 /(r w))^{2}} .
$$

For any $c$ on the unit circle

$$
S_{2 n}^{\prime} c-S_{2 n} n-S_{2 n} \frac{(r c)^{2}+1}{(r c)^{2}-1}=S_{2 n} *\left(D_{2 n}-\frac{(r c)^{2}+1}{(r c)^{2}-1} I_{2 n}\right)(c)
$$

Let us find the zeros of the Szegő composition (3.4). We have

$$
H(z)=D_{2 n}(z)-\frac{(r c)^{2}+1}{(r c)^{2}-1} I_{2 n}(z)=n(z+1)^{2 n-1}(z-1)-\frac{(r c)^{2}+1}{(r c)^{2}-1}(z+1)^{2 n} .
$$

The polynomial $H$ vanishes at the points $z_{1}=-1$ and $z_{2}=\frac{(r c)^{2}(n+1)-(n-1)}{(r c)^{2}(n-1)-(n+1)}$. Consider the Mőbius transformation $g(z)=\frac{z(n+1)-(n-1)}{z(n-1)-(n+1)}$. It is easy to see that $g$ maps the real line to the real line, $|g(0)|<1, g(-1)=1$ and $g(1)=-1$. Hence the image of the unit disk by $g$ is the unit disk. Therefore, $\left|z_{2}\right|=\left|g\left((r c)^{2}\right)\right|<1$ and all the zeros of $H$ lie in the closed unit disk. At each point on the unit circle expression (3.4) is equal to the Szegő composition of $S_{2 n}$ and the polynomial depending on this point and having all the zeros in the open unit disk. By the Szegö theorem 2.1, this composition can vanish only in the open unit disk. Therefore, the numerator of (3.3) (and (3.3) itself) does not vanish on the unit circle, consequently $f_{n}^{\prime \prime}(z)=M_{n}^{\prime \prime}(z)-\alpha p_{n}^{\prime \prime}(z) \neq 0$ for any $z \in E_{r}$ and $|\alpha|<1$. Using the same arguments as before, we obtain

$$
\left|p_{n}^{\prime \prime}(z)\right| \leq\left|M_{n}^{\prime \prime}(z)\right|, \quad z \in E_{r} .
$$

\section{DUFFIN-SCHAEFFER MAJORANT}

Consider the function

$$
M_{n}(z)=\frac{1}{r^{n}}\left(T_{n}(z)+\frac{1}{n} \sqrt{z^{2}-1} T_{n}^{\prime}(z)\right)=\frac{1}{r^{n}}\left(T_{n}(z)+\frac{i}{n} \sqrt{1-z^{2}} T_{n}^{\prime}(z)\right), \quad z \in E_{r},
$$

where $T_{n}$ is defined by (1.2). An easy computation shows that $M_{n}(z)=\frac{1}{r^{n}}\left(z+\sqrt{z^{2}-1}\right)^{n}$. By (2.1)

$$
M_{n}(G(r w))=\frac{1}{2 r^{n}}\left(r w+\frac{1}{r w}+r w-\frac{1}{r w}\right)^{n}=w^{n} .
$$

Thus $\left|M_{n}(z)\right|=1, z \in E_{r}$ and the function $M_{n}(z)$ satisfies condition (1.7) for the majorant in Theorem 1.6. Let us calculate $\left|M^{\prime}(z)\right|$ and $\left|M^{\prime \prime}(z)\right|$. Since $\left|z+\sqrt{z^{2}-1}\right|=r$, we get

$$
M_{n}^{\prime}(z)=\frac{1}{r^{n}}\left(\left(z+\sqrt{z^{2}-1}\right)^{n}\right)^{\prime}=\frac{n}{r^{n}} \frac{\left(z+\sqrt{z^{2}-1}\right)^{n}}{\sqrt{z^{2}-1}}, \quad\left|M_{n}^{\prime}(z)\right|=\frac{n}{\left|\sqrt{z^{2}-1}\right|},
$$




$$
\begin{gathered}
M_{n}^{\prime \prime}(z)=\left(\frac{n}{r^{n}} \frac{\left(z+\sqrt{z^{2}-1}\right)^{n}}{\sqrt{z^{2}-1}}\right)^{\prime} \\
=\frac{n}{r^{n}}\left(\frac{n\left(z+\sqrt{z^{2}-1}\right)^{n}}{\sqrt{z^{2}-1}} \frac{1}{\sqrt{z^{2}-1}}-\left(z+\sqrt{z^{2}-1}\right)^{n} \frac{z}{\left(z^{2}-1\right)^{3 / 2}}\right)=\frac{n}{r^{n}}\left(z+\sqrt{z^{2}-1}\right)^{n} \cdot \frac{n \sqrt{z^{2}-1}-z}{\left(z^{2}-1\right)^{3 / 2}} .
\end{gathered}
$$

Applying Theorem 1.6 we obtain the extension of the Duffin-Schaeffer inequality to the ellipse $E_{r}$ for the first and second derivatives. If $\left|p_{n}(z)\right| \leq\left|M_{n}(z)\right|=1, z \in E_{r}$, then

$$
\left|p_{n}^{\prime}(z)\right| \leq \frac{n}{\left|\sqrt{z^{2}-1}\right|}, \quad z \in E_{r}
$$

and

$$
\left|p_{n}^{\prime \prime}(z)\right| \leq n\left|\frac{n \sqrt{z^{2}-1}-z}{\left(z^{2}-1\right)^{3 / 2}}\right|, \quad z \in E_{r} .
$$

It is worth pointing out that inequality (4.3) is the Sewell pointwise inequality (1.6). Indeed, note that

$$
G\left(r e^{i t}\right)=\frac{1 / r+r}{2} \cos t-i \frac{1 / r-r}{2} \sin t, \quad t \in[0,2 \pi] .
$$

Using (2.1) and (4.4), we have

$$
\begin{aligned}
& \left|\sqrt{z^{2}-1}\right|=\frac{1}{2}\left|r e^{i t}-\frac{1}{r} e^{-i t}\right|=\frac{1}{2}|r \cos t+i r \sin t-1 / r \cos t+i / r \sin t| \\
= & \frac{1}{2} \sqrt{(1 / r-r)^{2} \cos ^{2} t+(1 / r+r) \sin ^{2} t}=\frac{1}{2} \sqrt{(1 / r+r)^{2}+(1 / r-r)^{2}-4|z|^{2}} .
\end{aligned}
$$

Substituting this in (4.3), we obtain inequality (1.6).

\section{VIDENSKY MAJORANT}

Consider a function

$$
f_{n}(z)=K_{n}(z)+i \sqrt{z^{2}-1} L_{n-1}(z), \quad z \in E_{r},
$$

where $K_{n}, L_{n-1}$ are algebraic polynomials of exact degrees $n$ and $n-1$ respectively. Using (2.1), it is easy to check that

$$
f_{n}(G(r w))=\frac{1}{w^{n}} \sum_{j=0}^{2 n} c_{j} w^{j}, \quad|w|=1 .
$$

By (4.4), it is clear that

$$
\tau_{n}(t)=f_{n}\left(G\left(r e^{i t}\right)\right)=f_{n}\left(\frac{1 / r+r}{2} \cos t-i \frac{1 / r-r}{2} \sin t\right), \quad \Re \tau(t), \quad \mathfrak{I} \tau(t)
$$

are trigonometric polynomials of exact degree $n$. Let $\Re \tau_{n}(t)$ and $\mathfrak{I} \tau_{n}(t)$ have $2 n$ distinct zeros lying on the period and separating one another. This implies that the curve $\tau_{n}(t)$ encircles the origin $n$ times, when $t$ changes from 0 to $2 \pi$. Let $M_{n}(z)=f_{n}(z)$, if the curve $\tau_{n}(t)$ is positively oriented. Otherwise let $M_{n}(z)=\overline{f_{n}(z)}$. In the second case we have the representation

$$
M_{n}(G(r w))=\frac{1}{w^{n}} \sum_{k=0}^{2 n} \bar{c}_{k} w^{2 n-k} .
$$

So, the winding number of $M_{n}$ around the origin is equal to $n$.

Let $s$ be the multiplicity of the root $w=0$ for the polynomial $Q_{2 n}$ corresponding to $M_{n}$. By the argument principle for

$$
M_{n}(G(r w))=\frac{Q_{2 n}(w)}{w^{n}}=\frac{\widetilde{Q}_{2 n}(w)}{w^{n-s}},
$$


the number of zeros of $M_{n}(G(r w))$ in the open unit disk is equal to the winding number of $M_{n}(G(r w))$ plus the number of its poles in this domain. Its winding number is equal to $n$, the number of poles is equal to $n-s$. Therefore, the number of zeros of $\widetilde{Q}_{2 n}$ in the open unit disk is equal to $2 n-s$, that is this value for $Q_{2 n}$ is equal to $2 n$.

Hence we may apply Theorem 1.6 in this case. For algebraic polynomials $p_{n}$ of degree $n$ such that

$$
\left|p_{n}(z)\right| \leq\left|M_{n}(z)\right|, \quad z \in E_{r},
$$

we obtain the extension of Vidensky's inequality to the ellipse $E_{r}$ for the first and second derivatives

$$
\left|p_{n}^{(k)}(z)\right| \leq\left|M_{n}^{(k)}(z)\right|, \quad z \in E_{r}, k=1,2 .
$$

REMARK 5.1. It can be proved that if all the zeros of the polynomial corresponding to $M_{n}^{(k)}$ lie in the open unit disk, then our method works for the derivative of order $k+1$. However, let us show that if the polynomials $K_{n}(z)$ and $L_{n-1}(z)$ have real coefficients, then the condition for the majorant's zeros is always violated for $k>2$.

Using (3.3) and (2.2), it is not hard to see that the following representation is valid:

$$
\frac{d^{2} M_{n}}{d z^{2}}(G(r w))=\frac{Q_{2 n+2}(w)}{w^{n+1}(r w-1 /(r w))^{3}}, \quad|w|=1,
$$

where $Q_{2 n+2}$ is an algebraic polynomial of degree $2 n+2$.

The Joukowsky transform maps $w$-plane on $z$-plane and $G(w) \in[-1,1], G(r w) \in E_{r}(0<r<1)$ for $|w|=1$. Since the real part of the majorant $M_{n}$ on $[-1,1]$ is an algebraic polynomial of degree $n$, the real part of $M_{n}^{(2)}$ is a polynomial of degree $n-2$. Hence the curve $M_{n}^{(2)}(G(w))(w-1 / w)^{3}$ encircles the origin at most $n-1$ times, when $w$ goes around the unit circle. Then, by the argument principle and $(5.1), M_{n}^{(2)}(G(w))(w-1 / w)^{3}$ has at most

$$
n-1+n+1=2 n
$$

zeros in the open unit disk. That is, the polynomial corresponding to $M_{n}^{(2)}(G(w))$ on the unit circle has at most $2 n$ zeros in the open unit disk. Hence we have this also for $M_{n}^{(2)}(G(r w))$. By (5.1), the degree of the polynomial corresponding to $M_{n}^{(2)}$ is equal to $2 n+2$, and the condition for the majorant's zeros is violated.

\section{ACKNOWLEDGEMENTS}

The author is grateful to P. Yu. Glazyrina and Sz. Gy. Révész for their attention to the work and valuable discussion.

The work was performed as part of the research conducted in the Ural Mathematical Center with the financial support of the Ministry of Science and Higher Education of the Russian Federation (Agreement number 075-02-2021-1383).

\section{REFERENCES}

[1] Bernstein, S. N. Sur l'ordre de la meilleure approximation des fonctions continues par des polynômes de degré donné. Mémoires de l'Académie royale de Belgique 4, 2 (1912).

[2] Bernstein, S. N. Leçons sur les Propriétés Extrémales et la Meilleure Approximation des Fonctions Analytiques d'une Variable Réelle. Paris, 1926.

[3] Bernstein, S. N. Sur la limitation des dérivées des polynômes. C. R. Acad. Sc. Paris 190 (1930), 338-340.

[4] De Bruijn, N. G. Inequalities concerning polynomials in the complex domain. Nederl. Akad. Wetensch. Proc. 50 (1947), 1265-1272.

[5] Duffin, R. J., And Schaeffer, A. C. On some inequalities of S. Bernstein and W. Markoff for derivatives of polynomials. Bull. Amer. Math. Soc. 44, 4 (1938), 289-297.

[6] Riesz, M. Eine trigonometrische Interpolationsformel und einige Ungleichungen für Polynome. Bull. Amer. Math. Soc. 44, 4 (1938), 289-297. 
[7] Sewell, W. E. On the polynomial derivative constant for an ellipse. Amer. Math. Monthly. 44 (1937), 895-903.

[8] SzEGő, G. Bemerkungen zu einem Satz von J. H. Grace über die Wurzeln algebraischer Gleichungen. Math. Z. 13 (1922), 28-55.

Open Access statement. This is an open-access article distributed under the terms of the Creative Commons AttributionNonCommercial 4.0 International License (https://creativecommons.org/licenses/by-nc/4.0/), which permits unrestricted use, distribution, and reproduction in any medium for non-commercial purposes, provided the original author and source are credited, a link to the CC License is provided, and changes - if any - are indicated. 